\section{Management of a young teenager with recurrent cough and a feeding problem}

\section{Case history}

A 12-year-old Malay female first presented to a district hospital with severe bronchopneumonia, which required ventilation for 28 days in March 2005.

She had experienced chronic intermittent cough since the age of 3 years, which became persistent by the age of 5 years. The cough was moist but was non-productive. There was also a history of feeding difficulties from early infancy. She took a long time to finish bottle feeds and tended to vomit when given a large amount. Although the weaning process started at 7 months, she was only completely weaned onto solid food by the age of 5 years. The patient complained of feeling full easily after eating. She denied any history of dysphagia, nocturnal regurgitation or abdominal pain.

At presentation, the patient was emaciated, with a mass of $13.8 \mathrm{~kg}$ ( $<3 \mathrm{rd}$ centile) and a height of $120 \mathrm{~cm}$ (<3rd centile). Gross finger clubbing was noted. She was tachypnoiec with mild chest recession. The trachea was deviated to the right, and the chest was deformed with depressed right hemithorax and thoracic convexity to the left. The right lung showed reduced chest expansion, as well as reduced breath sound. There was bronchial breathing at the right middle and lower lobes with coarse crepitations. No rhonchi were observed. She had hepatomegaly of $3 \mathrm{~cm}$ below subcostal margin. The remainder of the examination was unremarkable.

Chest radiography and high-resolution computed tomography (HRCT) scan of the thorax were performed and the results are shown in figures 1 and 2, respectively.

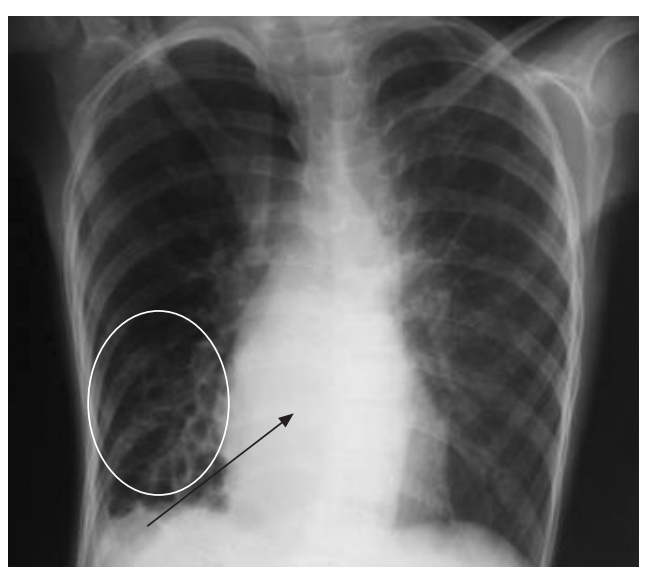

Figure 1

Chest radiograph.

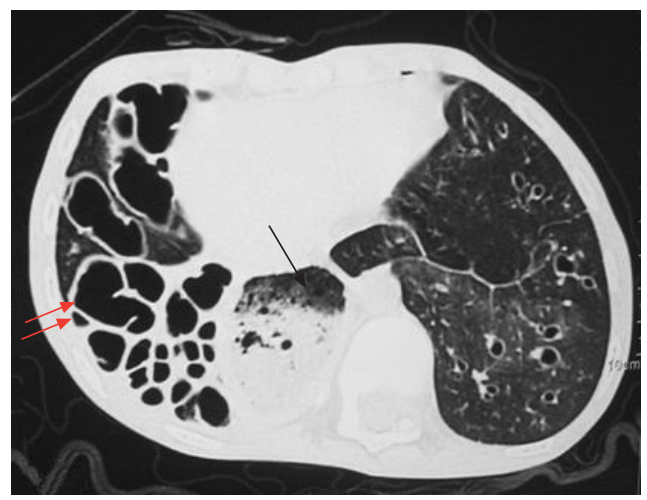

Figure 2

HRCT scan of the thorax.

\section{Task 1}

Interpret the chest radiograph and the HRCT scan.

\section{Task 2 \\ What investigations would you propose next?}

D. Mariana ${ }^{1}$

A. Rus Anida ${ }^{1}$

A.L. Hasniah ${ }^{1}$

A.M. Zaleha ${ }^{2}$

M. Zakaria 3

M.Z. Norzila ${ }^{1}$

Respiratory Unit, ${ }^{1}$ Dept of Paediatrics, ${ }^{2}$ Dept of Paediatric Radiology, and ${ }^{3}$ Dept of Paediatric Surgery, Paediatric Institute, Kuala Lumpur, Malaysia.

\section{Correspondence:}

D. Mariana

Respiratory Unit

Paediatric Dept

Paediatric Institute

Kuala Lumpur

Malaysia

E-mail:mdmariana@yahoo.com 


\section{Answer 1}

Figure 1 shows features of cystic bronchiectasis involving the right middle and lower zone (circled area) with bilateral lung hyperinflation and mild thoracic scoliosis. The distal oesophageal shadow is also dilated (arrow).

Figure 2 shows reduced right lung volume with cystic bronchiectatic changes (red arrows). The oesophagus is dilated and filled with food particles (black arrow). There are also signs of bronchial dilatation in the left lower lobe.

\section{Answer 2}

1. Barium swallow.

2. Oesophageal gastroduodenoscopy.

3. Oesophageal manometry.

A barium swallow was performed and is shown in figure 3. The barium swallow (figure 3) shows a grossly dilated oesophagus, and smooth tapering and narrowing of the distal lower oesophagus (arrow). There was delayed emptying of barium from the oesophagus to the stomach, which took about 2 hours. The stomach shadow was poorly distended with barium, suggesting a chronic under-usage of the stomach.

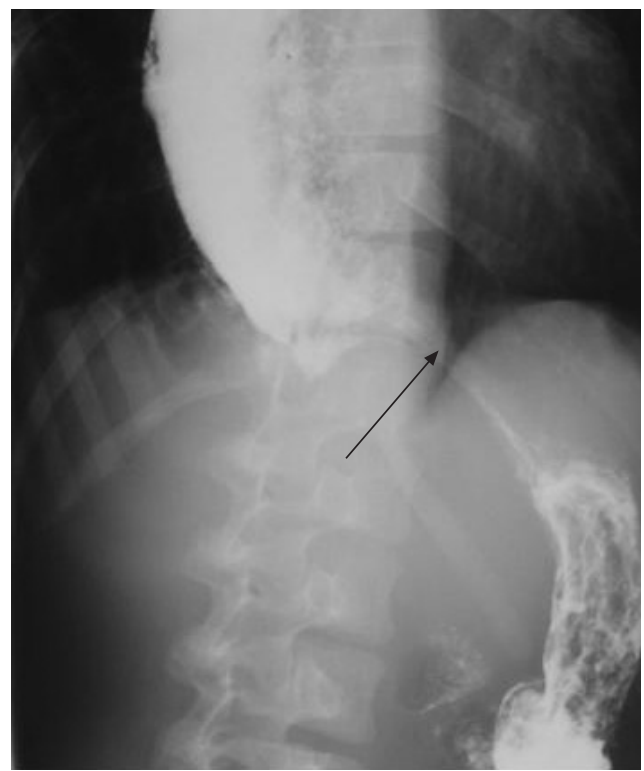

Figure 3

Barium swallow.
Upper endoscopic examination confirmed a constricted distal lower oesophageal junction; however, passage into the stomach was easy. In addition, no strictures were seen. The proximal oesophagus was severely dilated with a very thin wall (mega oesophagus). There was a moderate amount of food residually retained in the dilated oesophagus.

Oesophageal manometry could not be performed as no appropriately sized manometer was available. In fact, few paediatric centres are able to perform this procedure.

Other investigations to exclude other causes of bronchiectasis, including tuberculosis work-up, serum immunoglobulins, T- and B-cell subsets, and HIV screening, were all negative.

\section{Task 3 \\ Considering the information presented to you, what do you think is the most likely diagnosis?}

\section{Task 4 \\ Based on your diagnosis, how would you manage this patient?}




\section{Answer 3 \\ Cystic bronchiectasis secondary to oesophageal achalasia.}

\begin{abstract}
Answer 4
The patient was given total parenteral nutrition with a total calorie value of 120 $\mathrm{kcal} \cdot \mathrm{kg}^{-1} \cdot \mathrm{day}^{-1}$. She was advised to minimise aspiration, using methods such as minimal oral fluid intake and remaining in an upright position for at least 30 minutes to 1 hour after eating. Solid food or food with a high fibre content was not recommended. In order to treat the bronchiectasis, the patient was prescribed inhaled corticosteroid therapy and regular bronchodilator, in addition to regular chest physiotherapy and breathing exercises.
\end{abstract}

Open oesophageal cardiomyotomy with gastrostomy tube insertion was carried out in May 2005. Intra-operative findings confirmed the presence of oesophageal achalasia with mega oesophagus. The right lower lobe was removed in June 2005. The removed lobe was noted to be congested, bluish and non-expandable.

Post-operative recovery was uneventful. Since the operation, the patient can tolerate feeding without any symptoms of dysphagia or gastrooesophageal reflux. However, in view of the risk of aspiration, she was advised to only take small feeds. Additional nutritional supplement was given via gastrostomy. With this approach, her weight increased from $13.8 \mathrm{~kg}$ to $19.7 \mathrm{~kg}$ over a period of 12 weeks (see figure 4).

During follow-up, a repeat barium swallow was carried out 6 months post-surgery, which revealed only minimal improvement in terms of the radiological findings, although clinically the patient did not report any episodes of pneumonia.

\section{Discussion}

This young female presented with severe malnutrition and cystic bronchiectasis secondary to recurrent aspiration pneumonia due to oesophageal achalasia. The cause of the achalasia in this case is most probably idiopathic, as the symptoms had been present since early infancy, and investigations did not reveal secondary causes. Although investigations to exclude Chagas' disease were not carried out, the disease has never been reported in Malaysia.

Oesophageal achalasia is rare, with an estimated incidence of 0.11 cases per 100,000 of general population, and no more than $4-5 \%$ of

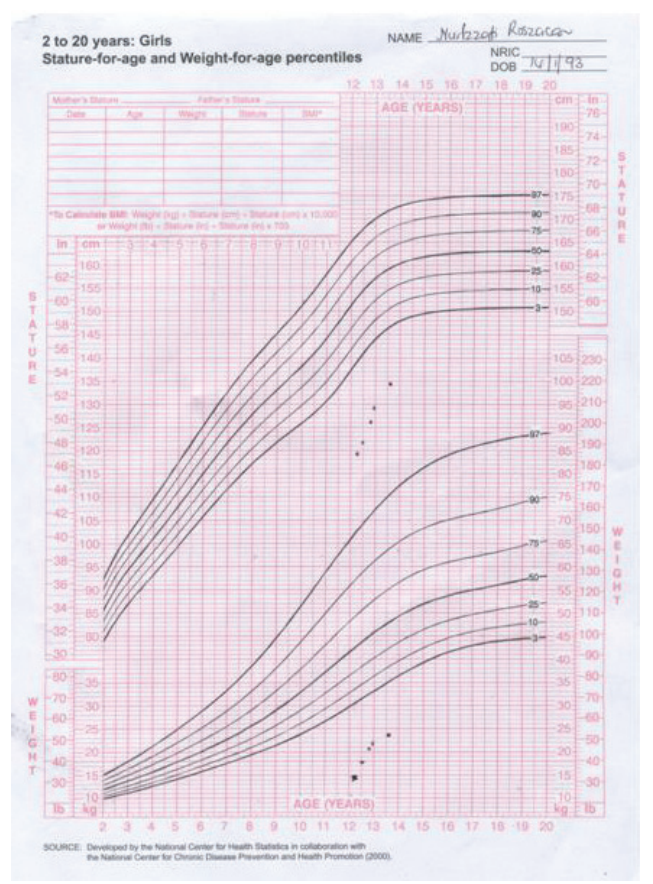

Figure 4

Centile chart. these cases have been described in children. Achalasia is an oesophageal motility disorder characterised by incomplete and uncoordinated relaxation of the lower oesophageal sphincter, associated with an aperistaltic oesophagus. The presentation varies from regurgitation and dysphagia to malnutrition and recurrent aspiration pneumonia [1-3]. Although recurrent pneumonia is a recognised presentation, bronchiectasis as a presenting complaint is rare.

In this case, the diagnosis of oesophageal achalasia was based on the presenting symptoms and initial chest radiographs, and was confirmed by a barium contrast study and upper endoscopy. In the barium contrast study, there was proximal oesophageal dilatation, with smooth tapering of the gastro-oesophageal junction. Endoscopic examination is important to confirm the presence of oesophagitis and other types of injury that are present with gastrooesophageal reflux disease, such as oesophageal stenosis. While oesophageal stenosis and strictures were excluded in the current patient, signs of chronic oesophagitis were observed, which were probably secondary to food stasis.

Oesophageal manometry is considered the examination of choice to confirm the presence of achalasia [2, 3]. Classically, there is increased lower oesophageal sphincter (LES) basal pressure, with partial or complete lack of relaxation of LES, and weak or absent peristaltic activity in the oesophagus [1-3]. However, for technical reasons, the investigation is rarely undertaken in children. 
The treatment of achalasia is generally directed at the symptomatic relief of functional obstruction. However, medical treatment has been extremely varied. Usually, only invasive modalities, such as pneumonic dilatation, surgery or botulinum toxin (botox) injection, have been shown to provide sustained benefit $[2$, 4-8]. The role of pneumatic dilatation or botox injection in children is still controversial, even though some data suggest that botox injection is an effective treatment approach in many patients with oesophageal achalasia, particularly when other therapeutic modalities have failed [7]. There are other treatment options, such as medication-based therapy with calcium channel blockers (e.g. nifedipine) [1], which act to relax the LES. However, in children, there is little information regarding benefits and side-effects of such treatment.

The standard treatment for oesophageal achalasia remains surgical and consists of cardiomyotomy with or without fundoplication $[1,2,4-6]$. Currently, laparoscopic Heller's cardiomyotomy with fundoplication is considered the treatment of choice in many centres $[1,4]$.

Management of the current patient was rather difficult, as she had already presented with several other severe complications (i.e. severe malnutrition, bronchiectasis and mega oesophagus). This allowed her nutritional status to be improved before proceeding to a right lobectomy.

As achalasia is an oesophageal motility disorder, surgery does not reverse the underlying pathology. Abnormal oesophageal peristalsis and a mega oesophagus persist, and the risk of aspiration remains high. Thus, careful monitoring and advice on appropriate feeding regimens are of paramount importance.

This case report describes a rare disorder leading to chronic pulmonary disease with severe bronchiectasis. If the chest radiograph or feeding history suggests an oesophageal disorder as the underlying cause, initial investigations should include a barium swallow and endoscopy. If achalasia is diagnosed, the patient should be monitored continuously for symptoms and signs of recurrent aspiration, even after successful surgery.

\section{References}

1. Marco Patti et al. Achalasia. 2004. http://www.emedicine.com/med/topic 16.htm

2. Sunny ZH, Ronald T, Vasundra T. A review of achalasia in 33 children. Digestive Diseases and Sciences. New York, Nov 2002; 47 (11): 2538-2543.

3. Fernandez PM, Lucio LA, Pollachi F. Esophageal achalasia of unknown etiology in children. J Pediatr 2004; 8: 523-526.

4. Patti MG, Albanese CT, Holcomb GW 3rd, et al. Laparoscopic Heller myotomy and Dor fundoplication for esophageal achalasia in children. J Pediatr Surg 2001; 36: 1248-1251.

5. Jun FL, Jun Z, Zi-Qiang T, et al. Long-term outcome of esophageal myotomy for achalasia. World J Gastroenterol 2004; 10: 287-291.

6. Oeschlager BK, Chang L, Pellegrini CA. Improved outcome after extended gastric myotomy for achalasia. Arch Surg 2003; 138: 490-497.

7. Annese V, Bassotti $G$, Coccia $G$, et al. A multicentre randomized study of intrasphincteric botulinum toxin in patient with oesophageal achalasia. GISMAD Achalasia Study Group. Gut 2000; 46: 597-600.

8. Hurwitz M, Bahar RJ, Ament ME, et al. Evaluation of the use of botulinum toxin in children with achalasia. J Pediatr Gastroenterol Nutr 2000; 30: 509-514. 